\title{
ON A CLASS OF STOKES FLOWS INSIDE A CORRUGATED BOUNDARY
}

\author{
BY \\ W. W. HACKBORN \\ Augustana University College, Camrose, Alberta, Canada
}

\begin{abstract}
A study is made of a family of steady two-dimensional Stokes flows stirred by an infinitesimal rotating cylinder (a line rotlet) inside a corrugated boundary. Parameters govern the position of the stirrer and the number and sharpness of ridges in the boundary. A solution is found in terms of a finite combination of elementary functions. It is demonstrated that the flow exhibits some interesting dynamical features such as peculiar bifurcations and homoclinic orbits.
\end{abstract}

1. Introduction. The stirring of a viscous fluid is an important component in many processes of interest in engineering and the natural sciences. In this paper, a model of steady two-dimensional stirring, at zero Reynolds number, of a viscous fluid inside a corrugated or furrowed boundary is considered. The stirring mechanism used is a line rotlet, a singularity which may be regarded as a rotating circular cylinder of infinitesimal radius. The boundary is modelled by an inverted (i.e., the geometric inverse of a) fluted column, an object that is easily constructed using the fluted column transformation of Godfrey [1] and the biharmonic inversion technique of Michell [2]. The position of the rotlet and the number and sharpness of ridges in the boundary can be varied. As will be seen, the solution to this problem can be expressed as a finite combination of elementary functions. Consequently, this solution can be computed quickly and accurately, and can more easily be made the basis of a higherorder analysis in which the perturbative effects of inertia or time-periodic forcing are introduced.

A number of previous studies have used a line rotlet as a stirrer. Ranger [3], for example, examined the Stokes flow due to a line rotlet inside a fixed or rotating circular cylinder. This flow is topologically equivalent to the flow between two nonconcentric circular cylinders (a journal bearing) induced by the rotation of the inner cylinder or both of the cylinders. (See Ballal and Rivlin [4] for a comprehensive analysis of the journal bearing flow.) However, the former flow has the advantage of being more tractable mathematically than the journal bearing flow. Hackborn, in [5] and [6], has also investigated the flow due to a line rotlet between fixed parallel planes and inside a fixed elliptic cylinder, respectively.

Received July 19, 1991.

1991 Mathematics Subject Classification. Primary 76.

(C)1993 Brown University 
The flow considered in this article is similar to flows studied by Ranger [7] and Pravica and Ranger [8]. Stokes flows stirred by a line rotlet and occurring in a generalized fluted column and the analytic inverse (with respect to a fixed inversion point) of an exterior fluted column are examined in [7] and [8], respectively. These flows exhibit attached eddies in some cases; however, the types of dynamical behaviour possible in these flows are limited by the facts that they are spatially periodic and the rotlet position cannot be varied. The flow examined in this paper exhibits some interesting dynamical features, such as peculiar bifurcations and interior saddle stagnation points, which are not present in the flows studied in [7] and [8]. Interior saddle stagnation points are very rare in Stokes flows driven by only one moving boundary. (The rotlet may be regarded as such a boundary.)

Aref [9] has noted that any incompressible two-dimensional flow may be regarded as a Hamiltonian dynamical system with one degree of freedom. In the theory of dynamical systems, interior saddle stagnation points (more commonly termed hyperbolic fixed points in the theory) play an important role. A hyperbolic fixed point possesses stable and unstable manifolds which, in a steady Hamiltonian system with one degree of freedom, either connect the point to another such point, producing a heteroclinic orbit, or connect the point to itself, producing a homoclinic orbit. A time-periodic perturbation of a steady flow possessing such orbits generally disrupts them causing heteroclinic or homoclinic tangles, a phenomenon indicative of nonintegrable or chaotic dynamics for passively advected particles in the flow. (See Lichtenberg and Lieberman [10] for an examination of chaos in Hamiltonian systems.) Aref and Balachandar [11] exploit this idea to generate chaos in a numerical simulation of a time-modulated version of the journal bearing flow. (The steady journal bearing flow, mentioned above, possesses either one or two hyperbolic fixed points depending on whether the cylinders are rotating in the opposite or in the same direction, respectively.) Chaiken et al. [12] and Swanson and Ottino [13], in combined experimental-numerical studies, have also examined the chaotic dynamics arising in time-modulated versions of the journal bearing flow. Aref [9] has moreover pointed out that chaotic dynamics produces efficient fluid stirring. Consequently, under a given time-periodic perturbation (e.g. resulting from a periodic motion of the rotlet), the flow considered in this article may exhibit a greater measure of chaos, and therefore enhanced stirring efficiency, for the cases in which the unperturbed flow possesses a hyperbolic fixed point. Hence, the results given in this paper might assist in the design of efficient stirring devices.

The problem to be investigated here is stated in Sec. 2 and solved in Sec. 3. In Sec. 4, a detailed analysis is made of the case in which the corrugated boundary containing the flow has a single ridge protruding into the flow region. Finally, some cases in which the boundary has more than one ridge are briefly considered in Sec. 5 .

2. Statement of the problem. The fluted column transformation (Godfrey [1]) is

$$
z=\zeta+c \zeta^{n+1}, \quad 0 \leq c \leq(n+1)^{-1},
$$

where $z$ and $\zeta$ are complex variables, and $n$ is a positive integer. Let $(\rho, \phi)$ be 
polar coordinates in the $\zeta$-plane (i.e., $\zeta=\rho e^{i \phi}$ ). The image in the $z$-plane of the unit circle $\rho=1$ in the $\zeta$-plane is a cross section of the fluted column. This cross section will be denoted by $F$. Note that $(2.1)$ has no critical points (i.e., points at which $d z / d \zeta=0)$ in the disk $\rho<1$. Godfrey [1] points out that $F$ lies in the annular region $1-c \leq|z| \leq 1+c$. Moreover, when $(n+1)^{-2}<c<(n+1)^{-1}, F$ is concave outward at the points satisfying $|z|=1-c$ [at which $\phi=\pi(2 m+1) / n, m=$ $0,1, \ldots, n-1]$; hence, for this interval of $c$ values, these points may be regarded as the peaks of $n$ ridges that protrude into the interior of $F$. If $c=(n+1)^{-1}$, the critical points of (2.1) coincide with these points, causing $F$ to have ridges with cusps at their peaks.

A geometric inversion of the $z$-plane with respect to the point $z=d$ (where $d$ is assumed to be real) is described by

$$
z_{i}=k(\bar{z}-d)^{-1}, \quad d<c(-1)^{n+1}-1 \text { or } d>c+1,
$$

where $z_{i}$ is complex, $k$ is a real scaling factor to be specified below, and the overbar denotes complex conjugation. The image in the $z_{i}$-plane of $F$ will be denoted by $F_{i} ; F_{i}$ is a cross section of the inverted fluted column. The condition on $d$ in (2.2) insures that the inversion point lies outside $F$ and, consequently, that (2.2) maps the interior of $F$ to the interior of $F_{i}$. Note also that (2.1) and (2.2) imply that $(\rho, \phi)$ form a left-handed, orthogonal, curvilinear coordinate system in the $z_{i}$-plane. In terms of Cartesian coordinates in the $z$ - and $z_{i}$-planes defined by $z=x+i y$ and $z_{i}=x_{i}+i y_{i},(2.2)$ becomes

$$
x_{i}=k(x-d) R^{-2}, \quad y_{i}=k y R^{-2}, \quad R=\left[(x-d)^{2}+y^{2}\right]^{1 / 2} .
$$

Let $I_{1}$ and $I_{2}$, with $I_{1}<I_{2}$, denote the $x_{i}$-intercepts of $F_{i}$. It will be assumed that the coordinates $\left(x_{i}, y_{i}\right)$ are dimensionless relative to the distance $I_{2}-I_{1}$. Therefore, the dimensionless scaling factor $k$ will be chosen so that $I_{2}-I_{1}=1$. Hence, using (2.1) and (2.3),

$$
k=\left\{\left[c(-1)^{n+1}-1-d\right]^{-1}-(c+1-d)^{-1}\right\}^{-1} .
$$

The flow region for the two-dimensional problem examined in this paper is the interior of $F_{i}$, the set of points in the $z_{i}$-plane for which $\rho<1$. The curves $F$ and $F_{i}$ are sketched in Fig. 1 (see p. 332) for the case $n=1, c=\frac{1}{2}$, and $d=-2$.

The flow to be studied will be described using a dimensionless stream function $\psi_{i}$ in terms of which the dimensionless fluid velocity $\mathbf{u}_{i}$ in the $z_{i}$-plane may be written as

$$
\mathbf{u}_{i}=\frac{R^{2}}{k\left|1+(n+1) c \zeta^{n}\right|}\left[\frac{1}{\rho} \frac{\partial \psi_{i}}{\partial \phi} \hat{\boldsymbol{\rho}}-\frac{\partial \psi_{i}}{\partial \rho} \hat{\boldsymbol{\phi}}\right]
$$

where $\hat{\rho}$ and $\hat{\phi}$ are unit vectors in the directions of increasing $\rho$ and $\phi$. The stream function for the Stokes flow satisfies the biharmonic equation,

$$
\nabla_{i}^{4} \psi_{i}=0, \quad \nabla_{i}^{2} \equiv \frac{\partial^{2}}{\partial x_{i}^{2}}+\frac{\partial^{2}}{\partial y_{i}^{2}} .
$$



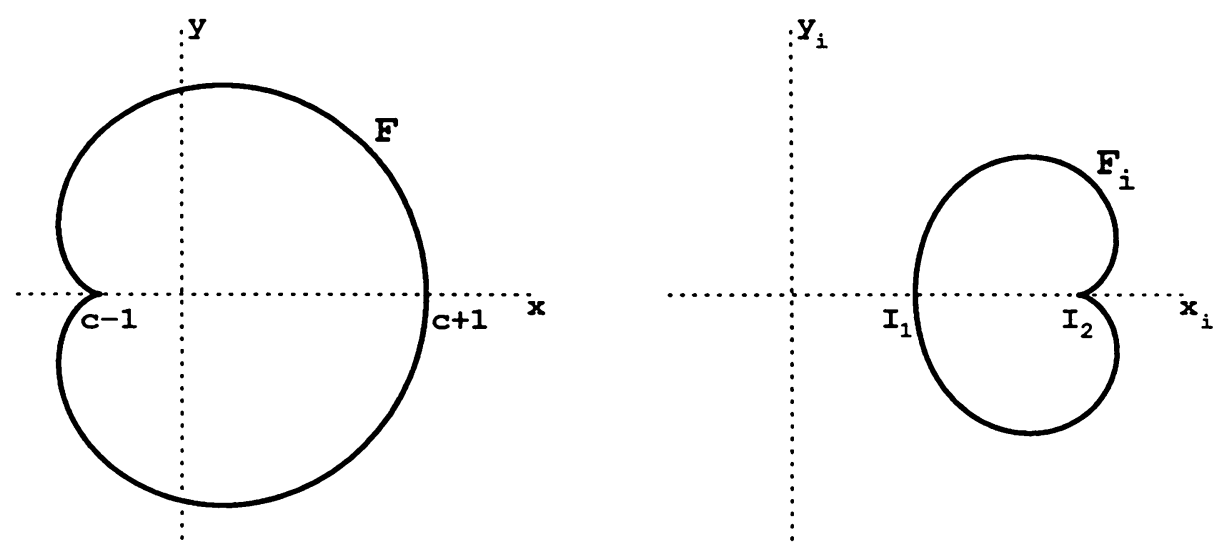

FIG. 1. The fluted column cross section $F$ and its geometric inverse $F_{i}$, with their $x$ - and $x_{i}$-intercepts labelled, for $n=1, c=\frac{1}{2}$, and $d=-2$.

The flow is driven by a line rotlet coinciding with $x_{i}=-k / d, y_{i}=0$ (at which $\rho=0$ ). A line rotlet, by definition, is a singularity that exerts a torque per unit length of magnitude $2 \Gamma \mu$ on the surrounding fluid, where $\Gamma$ is the circulation around the rotlet and $\mu$ is the viscosity of the fluid. Assuming that $\psi_{i}$ is dimensionless relative to $\Gamma /(2 \pi)$, the presence of the rotlet requires that

$$
\psi_{i}=\log R_{i}+\chi_{i}, \quad R_{i}=\left[\left(x_{i}+\frac{k}{d}\right)^{2}+y_{i}^{2}\right]^{1 / 2},
$$

where $\chi_{i}$ has no singularities in the flow region $\rho<1$. (Note that a line rotlet is mathematically equivalent to a line vortex of potential flow theory.) The no-slip condition on the boundary $F_{i}$ is satisfied if

$$
\psi_{i}=\frac{\partial \psi_{i}}{\partial \rho}=0 \quad \text { on } \rho=1 .
$$

3. Solution of the problem. Following the biharmonic inversion technique of Michell [2], the problem will be expressed in terms of a function $\psi$ defined by

$$
\psi=R^{2} \psi_{i}
$$

where $R$ is as in (2.3). Equation (2.6) now implies that $\psi$ is biharmonic with respect to $x$ and $y$, i.e.,

$$
\nabla^{4} \psi=0, \quad \nabla^{2} \equiv \frac{\partial^{2}}{\partial x^{2}}+\frac{\partial^{2}}{\partial y^{2}}
$$

and condition (2.8) may be expressed as

$$
\psi=\frac{\partial \psi}{\partial \rho}=0 \quad \text { on } \rho=1 .
$$

From (2.1), (2.3), and (2.7), it can be shown that

$$
\log R_{i}=\log \rho+\log \left|1+c \zeta^{n}\right|-\log R+\log |k / d| .
$$


Since all but the first term on the right-hand side of (3.4) are regular in the flow region $\rho<1,(3.1)$ and (3.4) imply that (2.7) may be written as

$$
\psi=R^{2} \log \rho+\chi,
$$

where $\chi$ has no singularities in the region $\rho<1$.

Since $\zeta$ and $z$ are related conformally by (2.1), the real part of any function analytic in $\zeta$ must be harmonic with respect to $x$ and $y$. Thus, $\log \rho$ is harmonic with respect to $x$ and $y$, and it is easily seen from this that $R^{2} \log \rho$ is biharmonic with respect to $x$ and $y$. Hence (3.2) and (3.5) imply that $\chi$ is also biharmonic with respect to $x$ and $y$. Now, (3.3) and (3.5) give

$$
\chi=0, \quad \frac{\partial \chi}{\partial \rho}=-R^{2} \quad \text { on } \rho=1 .
$$

Also, using (2.1) and (2.3),

$$
\begin{aligned}
R^{2}= & \rho^{2}+c^{2} \rho^{2 n+2}+d^{2}-2 d \rho \cos (\phi) \\
& +2 c \rho^{n+2} \cos (n \phi)-2 c d \rho^{n+1} \cos [(n+1) \phi] .
\end{aligned}
$$

From (3.7), it is clear that (3.6) can be satisfied by choosing $\chi$ to be an appropriate linear combination of the following biharmonic functions:

$$
\begin{gathered}
\rho^{m} \cos (m \phi), \quad m=0,1, n-1, n, n+1 ; \\
x \rho^{m} \cos (m \phi)+y \rho^{m} \sin (m \phi)=\rho^{m+1} \cos [(m-1) \phi]+c \rho^{n+m+1} \cos [(n-m+1) \phi], \\
m=1,2, n, n+1, n+2 .
\end{gathered}
$$

The expression in (3.8a) is harmonic (and hence biharmonic) with respect to $x$ and $y$ since $\rho^{m} \cos (m \phi)=\operatorname{Re}\left(\zeta^{m}\right)$. The expression in $(3.8 \mathrm{~b})$ is biharmonic with respect to $x$ and $y$ by virtue of the fact that if $\Omega$ is harmonic with respect to $x$ and $y$ then $x \Omega$ and $y \Omega$ are biharmonic. Taking $\chi$ to be a linear combination, with unspecified coefficients $A_{0}, A_{1}, \ldots, A_{9}$, of the functions given in (3.8) and rearranging yields

$$
\begin{aligned}
\chi= & A_{0}+A_{5} \rho^{2}+A_{8} c \rho^{2 n+2}+\left(A_{1} \rho+A_{6} \rho^{3}+A_{7} c \rho^{2 n+1}+A_{9} c \rho^{2 n+3}\right) \cos (\phi) \\
& +\left(A_{2} \rho^{n-1}+A_{6} c \rho^{n+3}+A_{7} \rho^{n+1}\right) \cos [(n-1) \phi] \\
& +\left(A_{3} \rho^{n}+A_{5} c \rho^{n+2}+A_{8} \rho^{n+2}\right) \cos (n \phi)+\left(A_{4} \rho^{n+1}+A_{9} \rho^{n+3}\right) \cos [(n+1) \phi]
\end{aligned}
$$

A system of linear equations for the unknown coefficients is obtained from (3.6), (3.7), and (3.9). A solution of this system is

$$
\begin{aligned}
& A_{0}=\left(1-c^{2}\right) P+1, \quad A_{1}=\left(2 c^{2}-1\right) Q-c^{2} d, \\
& A_{2}=c Q, \quad A_{3}=c, \quad A_{4}=-c d, \quad A_{5}=-P-1 \text {, } \\
& A_{6}=Q, \quad A_{7}=-2 c Q, \quad A_{8}=c P, \quad A_{9}=c d,
\end{aligned}
$$

where

$$
P=\frac{c^{2}+d^{2}-1}{2\left[1-(n+1) c^{2}\right]}, \quad Q=\frac{d\left[1-(n+1) c^{2}\right]}{1-2 n c^{2}} .
$$

The stream function $\psi_{i}$ is now completely specified in terms of the coordinates $(\rho, \phi)$ by (3.1), (3.5), (3.7), (3.9), and (3.10). 
4. Description of the flow for $n=1$. In this section, we will denote the points at which $F_{i}$ intersects the $x_{i}$-axis by $P_{1}$ and $P_{2}$. (The $x_{i}$-coordinates of these points were termed $I_{1}$ and $I_{2}$ above. Recall that $k$ was chosen so that $I_{2}-I_{1}=1$.) The $(\rho, \phi)$ coordinates of $P_{1}$ and $P_{2}$ are $(1,0)$ and $(1, \pi)$ respectively. The distance between $P_{1}$ and the rotlet (at $x_{i}=-k / d, y_{i}=0$ ) will be denoted by $D$. Hence, using (2.1) with $n=1,(2.3)$ and (2.4),

$$
D=-\frac{k}{d}-I_{1}=\left(\frac{1+c}{2}\right)\left(1+\frac{1-c}{d}\right) .
$$

Recalling from (2.2) that $d \in(-\infty, c-1) \cup(c+1, \infty)$ for $n=1$, it is clear from (4.1) that as $d$ increases in this domain, the value of $D$ decreases through the range $(0,(1+c) / 2) \cup((1+c) / 2,1)$. Hence, for any permissible value of $c$ (i.e., for $0 \leq c \leq \frac{1}{2}$ from (2.1)), the rotlet takes each position on the $x_{i}$-axis between $P_{1}$ and $P_{2}$ at a unique value of $d$ (if the limiting value $|d| \rightarrow \infty$ is allowed). Note, however, that as $d$ varies with $c$ fixed, both the rotlet position and the shape of the boundary $F_{i}$ vary; rotlet position and boundary shape cannot be varied independently. By inspecting the curvature of the boundary at $P_{2}$, it can be seen that, for $\frac{1}{4}<c<\frac{1}{2}$ and $|d|$ sufficiently large, $F_{i}$ has a smooth ridge centered at $P_{2}$ that protrudes into the flow region. Moreover, when $c=\frac{1}{2}, F_{i}$ has a ridge with a sharp cusp at $P_{2}$ for any value of $d$.

Since $\psi, \psi_{\rho}, \psi_{\phi}, \psi_{\rho \phi}$, and $\psi_{\phi \phi}$ all vanish on the boundary $\rho=1$, the sign of $\psi$ [and hence $\psi_{i}$ by (3.1)] near the boundary is determined by the sign of $\psi_{\rho \rho}$. (Greek subscripts denote partial differentiation here.) Eddies attached to the boundary occur in simply-connected regions in which $\psi_{i}$ exists and has constant sign; these eddies are separated from adjacent regions by dividing streamlines (or separatrices) along which $\psi_{i}=0$ and which intersect the boundary at points (termed separation points) where $\psi_{\rho \rho}=0$. Now, from (3.5), (3.7), and (3.9), it is readily seen that $\psi_{\rho \rho}$ at $\rho=1$ is quadratic in $\cos (\phi)$; so there are at most four separation points, two for each root of this quadratic between -1 and 1 .

Computations and local analyses of the flow near $P_{1}$ and $P_{2}$ reveal that for permissible values of $d$ lying sufficiently close to $c+1$ or $c-1$, or equivalently for $D$ values sufficiently close to 1 or 0 , the flow has a single attached (i.e., attached to the boundary) eddy adjacent to $P_{1}$ or $P_{2}$, respectively. Such an eddy appears in Fig. 2(a). There are no eddies in the flow if and only if $D$ lies in a certain closed subinterval of the interval $(0,1)$; as $c \rightarrow \frac{1}{2}^{-}$, this subinterval decreases in length, and, when $c=\frac{1}{2}$, there are eddies for all values of $D$ except $D=\frac{3}{4}$ (i.e., except in the limiting case of $|d| \rightarrow \infty$, as will be shown in the next section). When $d>c+1$, at most one eddy exists; as mentioned above, one attached eddy adjacent to $P_{1}$ exists if $d$ is sufficiently close to $c+1$, and, as $d$ increases, this eddy shrinks and eventually vanishes unless $c=\frac{1}{2}$ in which case the eddy vanishes only in the limit as $d \rightarrow \infty$. On the other hand, when $d<c-1$, the flow is much more interesting; in this case, the flow can exhibit two attached eddies, as in Figs. 2(b) and 2(d), or two "free" eddies bounded by a double homoclinic orbit (figure-eight), as in Fig. 2(c).

The interesting bifurcations that can occur in the flow as $d$ decreases in the interval 


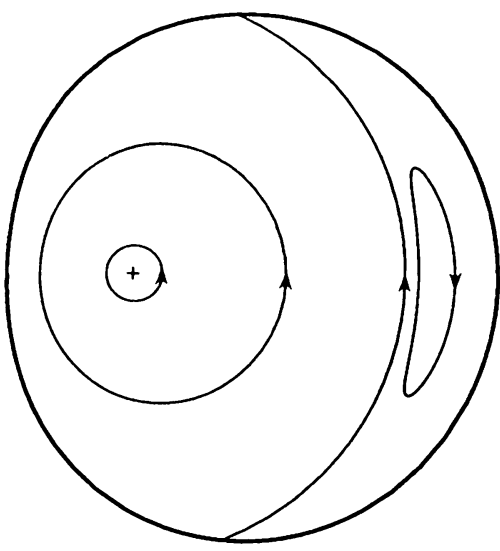

(a)

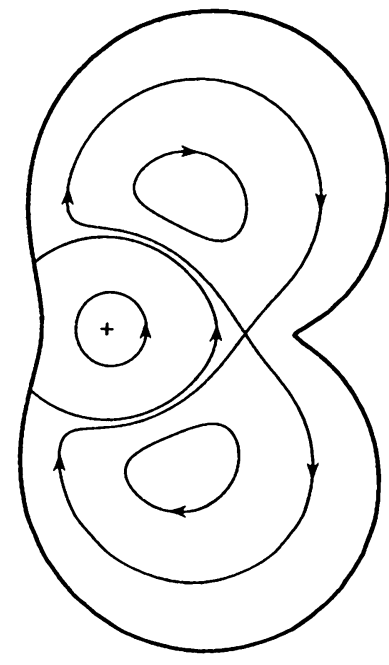

(c)

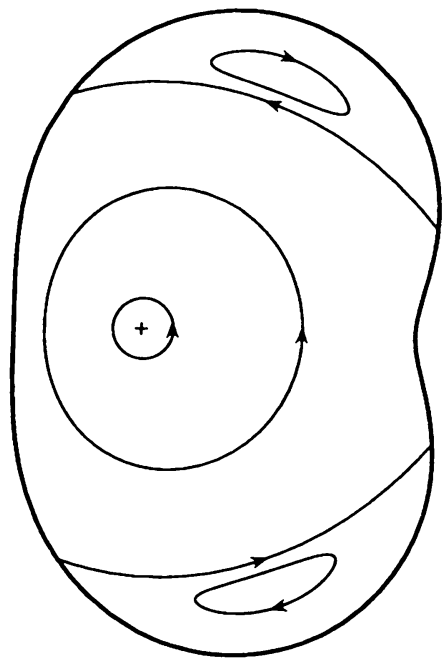

(b)

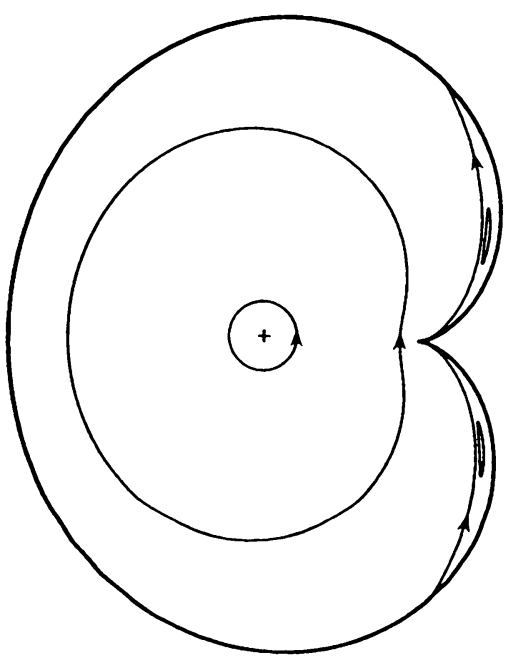

(d)

Fig. 2. Exact streamlines of the flow for $n=1$ and (a) $c=0.2$, $d=-1.4$, (b) $c=0.4, d=-1.1$, (c) $c=0.48, d=-0.8$, (d) $c=\frac{1}{2}, d=-3$. The position of the rotlet is indicated by "+".

$(-\infty, c-1)$ while $c$ is held fixed will now be explored. The basic tool of this exploration is the Taylor expansion of $\psi$ about $P_{2}$ :

$$
\begin{aligned}
\psi(\rho, \phi)= & \frac{1}{2} \psi_{\rho \rho}(1, \pi)(\rho-1)^{2}+\frac{1}{6} \psi_{\rho \rho \rho}(1, \pi)(\rho-1)^{3} \\
& +\frac{1}{4} \psi_{\rho \rho \phi \phi}(1, \pi)(\rho-1)^{2}(\phi-\pi)^{2}+\frac{1}{24} \psi_{\rho \rho \rho \rho}(1, \pi)(\rho-1)^{4}+\cdots
\end{aligned}
$$


All nonzero terms up to and including those of order four are given in (4.2). Since the term involving $\psi_{\rho \rho}(1, \pi)$ is normally the dominant term in (4.2), bifurcations can occur at values of $d$ at which $\psi_{\rho \rho}(1, \pi)$ changes sign. Now, if $0 \leq c<\frac{1}{2}$, $\psi_{\rho \rho}(1, \pi)$ is quadratic in $d$, and, if $c=\frac{1}{2}, \psi_{\rho \rho}(1, \pi)$ vanishes for all parameter values; moreover, when $0 \leq c<\frac{1}{2}$, one of the roots of the quadratic expression lies in the interval $(-\infty, c-1)$ and the other in the interval $(c-1, c+1)$. Let $d_{b}(c)$ denote, for $0 \leq c<\frac{1}{2}$, the lesser of these roots or, for $c=\frac{1}{2}$, the limit of this root as $c \rightarrow \frac{1}{2}^{-}$. Using (3.5), (3.7), (3.9), and (3.10) yields

$$
d_{b}(c)=\frac{-2 c^{3}+2 c^{2}+c-1-\left(4 c^{6}-8 c^{5}+4 c^{4}+8 c^{3}-4 c^{2}+2\right)^{1 / 2}}{2 c+1} .
$$

For $d_{b}(c)<d<c-1, \psi_{\rho \rho}(1, \pi)>0$ if $0 \leq c<\frac{1}{2}$, and $\psi_{\rho \rho}(1, \pi)=0$, $\psi_{\rho \rho \rho}(1, \pi)<0$ and $\psi_{\rho \rho \phi \phi}(1, \pi)>0$ if $c=\frac{1}{2}$. Hence, from $(4.2), \psi>0$ in the flow region $\rho<1$ sufficiently close to $P_{2}$ when $d_{b}(c)<d<c-1$ and $0 \leq c \leq \frac{1}{2}$; thus, since $\psi<0$ near the rotlet, there is an attached eddy adjacent to $P_{2}$ for these parameter values, and numerical work shows that this is the only attached eddy in the flow (although two free eddies may exist inside this attached eddy). The bifurcations that occur as $d$ decreases through $d_{b}(c)$ while holding $c$ fixed depend primarily on the signs of $\psi_{\rho \rho \rho}(1, \pi)$ and $\psi_{\rho \rho \phi \phi}(1, \pi)$ when $d=d_{b}(c)$. Computations show that, when $d=d_{b}(c), \psi_{\rho \rho \phi \phi}(1, \pi)<0$ for $0 \leq c<c^{*}$ and $\psi_{\rho \rho \phi \phi}(1, \pi)>0$ for $c^{*}<c \leq \frac{1}{2}$, while $\psi_{\rho \rho \rho}(1, \pi)>0$ for $0 \leq c<c^{* *}$ and $\psi_{\rho \rho \rho}(1, \pi)<0$ for $c^{* *}<c<\frac{1}{2}$, where $c^{*} \approx 0.2356$ and $c^{* *} \approx 0.4370$. There are now six cases to consider. [Unless otherwise stated, the assertions below follow from (4.2) or careful numerical work.]

(i) $0 \leq c<c^{*}$ : When $d \leq d_{b}(c), \psi<0$ in the flow region sufficiently close to $P_{2}$. Hence, the attached eddy adjacent to $P_{2}$ that exists for $d_{b}(c)<d<c-1$ vanishes as $d$ decreases through $d_{b}(c)$. Moreover, no eddies of any kind exist for $d \leq d_{b}(c)$.

(ii) $c=c^{*}$ : When $d=d_{b}(c), \psi_{\rho \rho \phi \phi}(1, \pi)=0$ but $\psi_{\rho \rho \phi \phi \phi \phi}(1, \pi)<0$; thus, $\psi<0$ in the flow region sufficiently close to $P_{2}$ when $d \leq d_{b}(c)$. This case is qualitatively identical to case (i).

(iii) $c^{*}<c<c^{* *}$ : When $d=d_{b}(c)$, a separatrix, on which $\psi=0$, lies in the flow region $\rho<1$ and is tangent to the boundary at $P_{2}$ near which it is described approximately by

$$
\rho-1=-\frac{3}{2}\left[\psi_{\rho \rho \phi \phi}(1, \pi) / \psi_{\rho \rho \rho}(1, \pi)\right](\phi-\pi)^{2} .
$$

Hence, as $d$ decreases through $d_{b}(c)$, the attached eddy that exists for $d_{b}(c)<d<$ $c-1$ "splits"; this results in two smaller eddies which shrink and eventually vanish as $d$ continues to decrease. Also, as $d \rightarrow d_{b}(c)^{+}$, the fixed point inside the attached eddy changes from elliptic (where $\psi_{i}$ has a maximum) to hyperbolic (where $\psi_{i}$ has a saddle), and the two free eddies bounded by the homoclinic orbits associated with the hyperbolic fixed point grow until they fill the entire attached eddy when $d=d_{b}(c)$. (The existence and growth of the free eddies is required by the continuity of the flow 


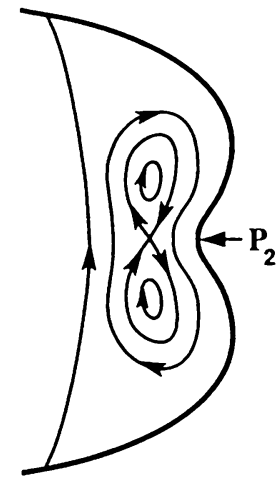

(a)

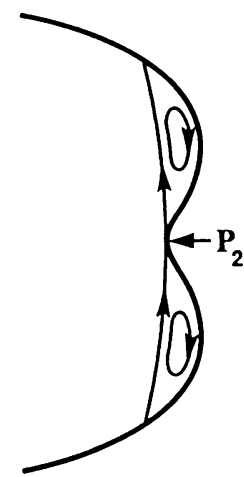

(b)

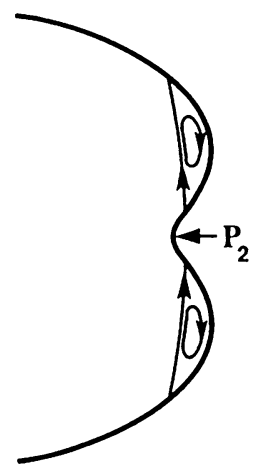

(c)

Fig. 3. Schematic sketches of streamlines near $P_{2}$ for $n=1$, $c^{*}<c<c^{* *}$ and (a) $d$ greater than and sufficiently close to $d_{b}(c)$, (b) $d=d_{b}(c),\left(\right.$ c) $d$ less than and sufficiently close to $d_{b}(c)$.

with respect to variations in a parameter near a value at which an attached eddy splits.) Figure 3 depicts the flow schematically for values of $d$ near $d_{b}(c)$.

(iv) $c=c^{* *}$ : When $d=d_{b}(c), \psi_{\rho \rho \rho}(1, \pi)=0$ but $\psi_{\rho \rho \rho \rho}(1, \pi)<0$; two parts of a separatrix, on which $\psi=0$, meet at a sharp corner at $P_{2}$, making an angle of $60^{\circ}$ with each other and the boundary. Otherwise, this case is qualitatively identical to case (iii).

(v) $c^{* *}<c<\frac{1}{2}$ : Sufficiently close to $P_{2}$ in the flow region $\rho<1, \psi>0$ when $d=d_{b}(c)$, but $\psi<0$ when $d<d_{b}(c)$. Thus, a small attached eddy begins to grow at $P_{2}$ beside the existing larger attached eddy as $d$ decreases through $d_{b}(c)$. As $d$ continues to decrease, the separatrices bounding these eddies eventually touch, splitting the larger eddy into two smaller ones; let $d_{s}(c)$ denote the value of $d$ at which this occurs. Free eddies like those of case (iii) develop as $d \rightarrow d_{s}(c)^{+}$, and, for $d$ values less than and sufficiently close to $d_{s}(c)$, the hyperbolic fixed point persists and is associated with a tiny free eddy. Figure 4 depicts the flow schematically for values of $d$ near $d_{s}(c)$. It should be noted that the flow studied here is the first example (known to this author) of a Stokes flow with an eddy at the peak of a smooth ridge. This eddy is very small; e.g., for $c=0.48$ and $d=-0.8688$, its width is about $0.3 \%$ of the distance between $P_{1}$ and $P_{2}$; Fig. 5 accurately shows all the separatrices (delimiting the attached and the free eddies) and the boundary near $P_{2}$ for these parameter values. Also, $d_{b}(c)$ and $d_{s}(c)$ differ by very little; e.g., $d_{b}(0.48) \approx-0.8683, d_{s}(0.48) \approx-0.8689$.

(vi) $c=\frac{1}{2}:$ In this case, $\psi_{\rho \rho}(1, \pi)$ vanishes for all values of $d$. Moreover, when $d=d_{b}(c), \psi_{\rho \rho \rho}(1, \pi)=\psi_{\rho \rho \rho \rho}(1, \pi)=0$, but $\psi_{\rho \rho \rho \rho \rho}(1, \pi)>0$ and $\Psi_{\rho \rho \phi \phi}(1, \pi)>$ 0 ; this implies that a separatrix, on which $\psi=0$, has a cusp at $P_{2}$ (where the boundary itself has a cusp oriented in the opposite direction) and the attached eddy that exists for $d_{b}(c)<d<c-1$ splits into two smaller ones as $d$ decreases through $d_{b}(c)$. Free eddies like those of case (iii) develop as $d \rightarrow d_{b}(c)^{+}$. When $d<d_{b}(c)$, $\psi_{\rho \rho \rho}(1, \pi)>0$, and $\psi_{\rho \rho \phi \phi}(1, \pi)>0$; hence, a separatrix, described approximately 


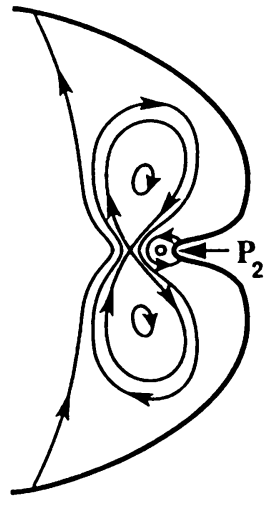

(a)

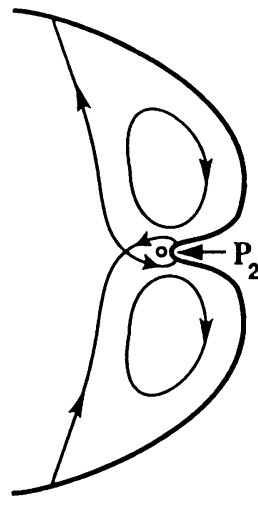

(b)

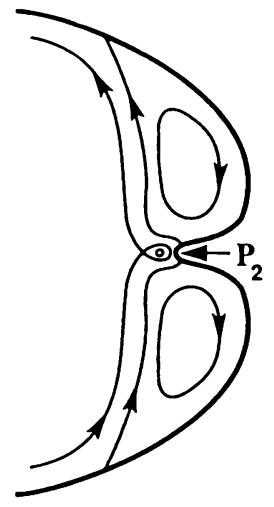

(c)

FIG. 4. Schematic sketches of streamlines near $P_{2}$ for $n=1, c^{* *}<$ $c<\frac{1}{2}$ and (a) $d_{s}(c)<d<d_{b}(c)$, (b) $d=d_{s}(c)$, (c) $d$ less than and sufficiently close to $d_{s}(c)$.

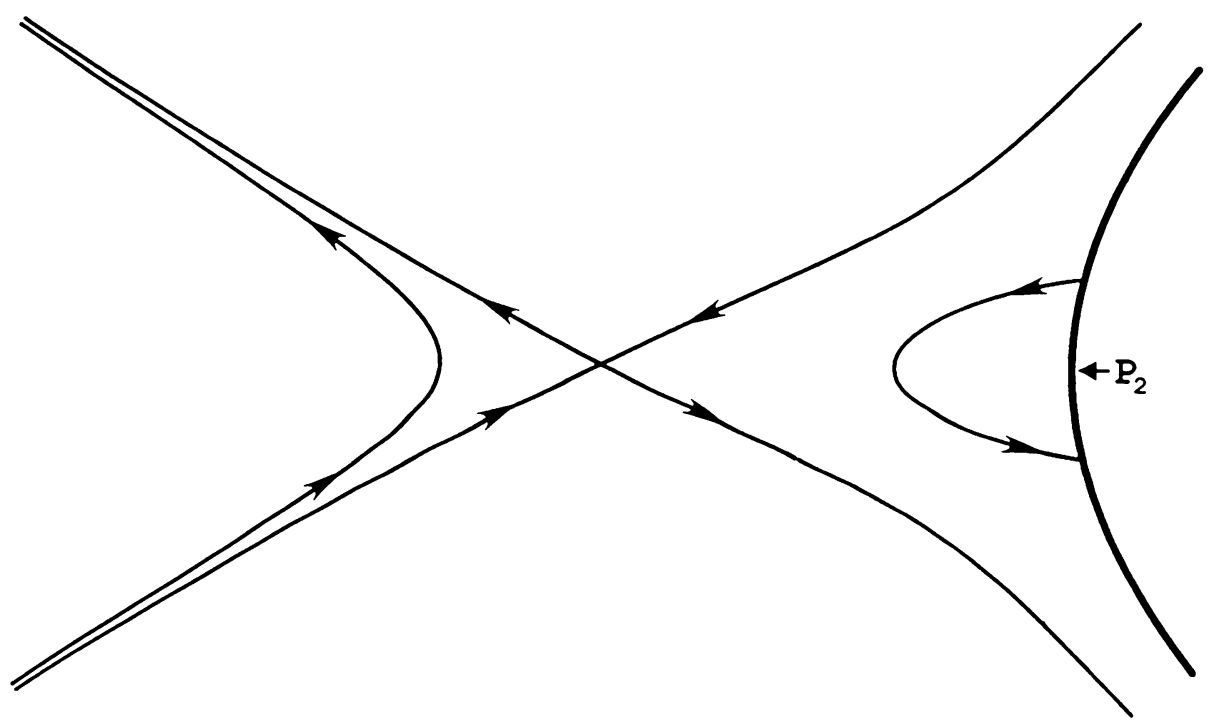

Fig. 5. Exact plots of all the separatrices (for both the attached and the free eddies) and the boundary near $P_{2}$ for $n=1, c=0.48$, and $d=-0.8688$.

by (4.4) near $P_{2}$, lies in the flow region $\rho<1$ and is tangent to the cusp of the boundary at $P_{2}$ [as in Fig. 2(d)]. The existence of this separatrix implies that eddies exist on each side of $P_{2}$ for all values of $d$ less than $d_{b}(c)$. These eddies shrink as $d$ decreases and vanish in the limit as $d \rightarrow-\infty$.

5. The general flow. As in the previous section, the points at which $F_{i}$ intersects the $x_{i}$-axis will be denoted here by $P_{1}$ and $P_{2}$, and $D$ will signify the distance 
between $P_{1}$ and the rotlet. From (2.1), (2.3), and (2.4),

$$
D=\left(\frac{2+\left[1-(-1)^{n}\right] c}{4}\right)\left(1+\frac{1+(-1)^{n} c}{d}\right) \text {. }
$$

When $n$ is odd, (5.1) reduces to (4.1). Now, the allowed values of $c$ and $d$ are given in (2.1) and (2.2), and suppose that the limit $|d| \rightarrow \infty$ is one possible value of $d$. Then, for any value of $c,(5.1)$ implies that $D$ takes each value between 0 and 1 (and so the rotlet takes each position between $P_{1}$ and $P_{2}$ ) at some unique value of $d$. However, as noted in Sec. 4, rotlet position and boundary shape cannot be varied independently. For $(n+1)^{-2}<c<(n+1)^{-1}$ and $|d|$ sufficiently large, $F_{i}$ has $n$ smooth ridges that protrude into the flow region, and, when $c=(n+1)^{-1}$, $F_{i}$ has $n$ ridges with cusps at their peaks for any value of $d$. [These cusps occur at $\phi=\pi(2 m+1) / n, m=0,1, \ldots, n-1$.]

The special case of $|d| \rightarrow \infty$ is worth examining. In this limiting case, the flow becomes equivalent to the flow in the $z$-plane inside the standard (i.e., not inverted) fluted column $F$ due to a rotlet at the origin since, as $|d| \rightarrow \infty$, transformation (2.3) uniformly approaches the composition of an isotropic dilation, a reflection in the $y$-axis, and a translation parallel to the $x$-axis. (This can be seen approximately even in Fig. 1 where $d=-2$.) Now, the uniform limit of $\psi_{i}$ as $|d| \rightarrow \infty$ is

$$
\lim _{|d| \rightarrow \infty} \psi_{i}=\log \rho+\frac{1-\rho^{2}-c^{2}\left(1-\rho^{2 n+2}\right)}{2\left[1-(n+1) c^{2}\right]} .
$$

Since the limiting stream function in (5.2) is independent of $\phi$, it represents a flow for which $\rho$ is constant on any streamline; hence, no eddies can exist. This result was also demonstrated by Ranger [7] in his analysis of flow in a generalized fluted column.

Another noteworthy case is that for which $c=0$. In this case, both the fluted column and its inverse are circular cylinders. In [3], Ranger examined the flow due to a line rotlet inside a circular cylinder; it was found that, for a cylinder of unit radius, a single eddy exists if and only if the distance between the rotlet and the cylinder's axis exceeds $\sqrt{2}-1$. This result is also true of the flow considered in this paper when $c=0$.

As stated in Sec. 4, separation points on the boundary $\rho=1$ can occur only where $\psi_{\rho \rho}$ vanishes. Since (3.5), (3.7), and (3.9) imply that $\psi_{\rho \rho}$ at $\rho=1$ is a polynomial of degree $n+1$ in $\cos (\phi)$, it is clear that the flow has at most $2(n+1)$ separation points. In Fig. 6 (see p. 340), the flow is depicted for $n$ varying from 2 to 5 and the values of $c$ and $d$ chosen (partly) so that in each case the boundary has $n$ ridges protruding into the flow region. Generally, attached eddies are more likely to form as the sharpness of the ridges increases (i.e., as $c$ approaches $(n+1)^{-1}$ from the left). This is illustrated well by Fig. 6(c) in which three eddies are visible; in fact, there are two more eddies, too thin to be visible, near the ridges farthest to the right in the figure; for slightly larger $c$ values, these thin eddies join with the visible thin eddies so that only three eddies exist. [Thus, the flow shown in Fig. 6(c) has ten separation points, the largest number possible for $n=4$.] When $c=(n+1)^{-1}$, separatrices 


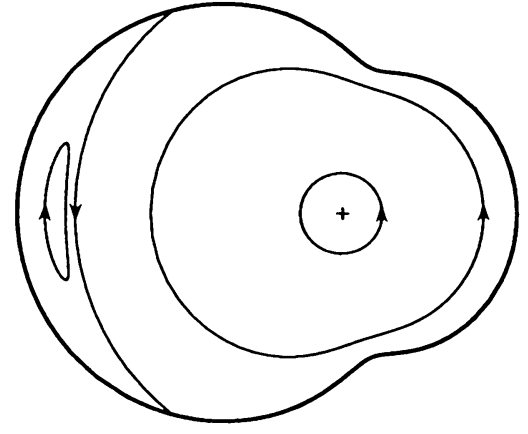

(a)

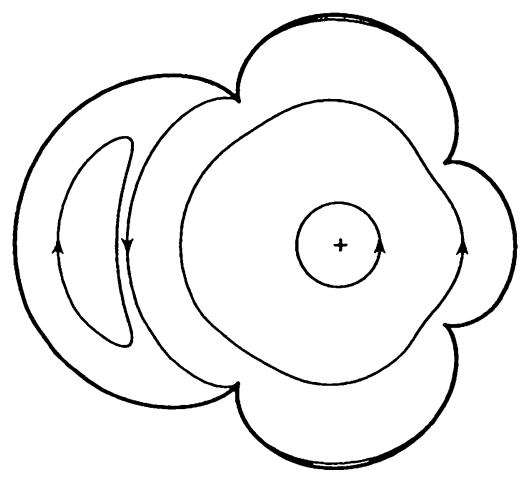

(c)

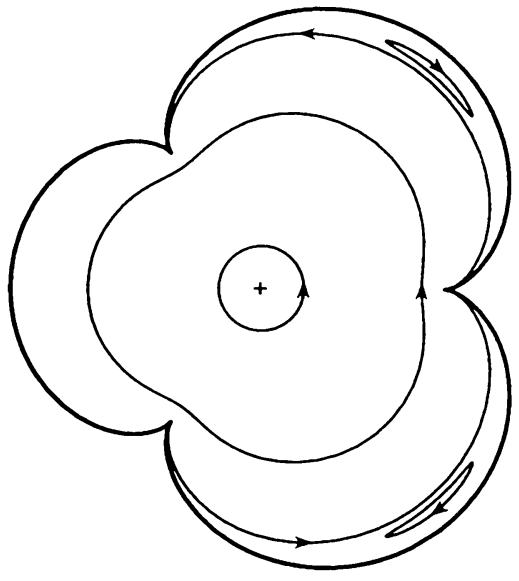

(b)

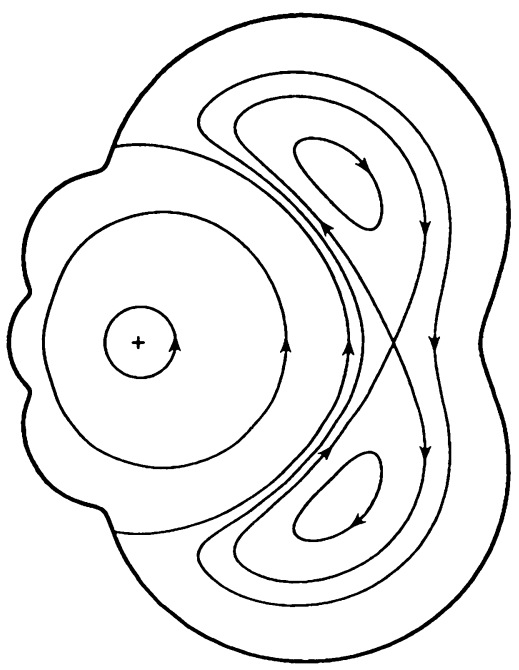

(d)

Fig. 6. Exact streamlines of the flow for (a) $n=2, c=0.2, d=4$, (b) $n=3, c=0.25, d=-10$, (c) $n=4, c=0.198, d=4$, (d) $n=5, c=0.1, d=-1.8$. The position of the rotlet is indicated by " + ".

delimiting attached eddies are often tangent to one or both sides of some or all of the cusps in the boundary, as in Fig. 6(b). Fig. 6(d) depicts a flow in which two free eddies, like those discussed in Sec. 4 , are bounded by a double homoclinic orbit associated with a hyperbolic fixed point. When $n>1$, it is possible for more than one hyperbolic fixed point to occur, but they lie very close to the boundary in all cases of this examined by the author. 
Acknowledgment. The author is grateful to the Center for Applied Mathematics at Cornell University, where he spent a sabbatical year during 1990-1991, for the use of their computing facilities in the production of the graphics that appear in this article.

\section{REFERENCES}

[1] D. E. R. Godfrey, Theoretical elasticity and plasticity for engineers, Thames and Hudson, London, 1959, pp. 58-59

[2] J. H. Michell, On the inversion of plane stress, Proc. London Math. Soc. 34, 142-229 (1901/02)

[3] K. B. Ranger, Eddies in two dimensional Stokes flow, Internat. J. Engrg. Sci. 18, 181-190 (1980)

[4] B. Y. Ballal and R. S. Rivlin, Flow of a Newtonian fluid between eccentric rotating cylinders: inertial effects, Arch. Rational Mech. Anal. 62, 237-294 (1976)

[5] W. W. Hackborn, Asymmetric Stokes flow between parallel planes due to a rotlet, J. Fluid Mech. 218, 531-546 (1990)

[6] W. W. Hackborn, Separation in a two-dimensional Stokes flow inside an elliptic cylinder, J. Engrg. Math. 25, 13-22 (1991)

[7] K. B. Ranger, Separation of streamlines for spatially periodic flow at zero Reynolds numbers, Quart. Appl. Math. 47, 367-373 (1989)

[8] D. W. Pravica and K. B. Ranger, Spatially periodic Stokes flow stirred by a rotlet interior to a closed corrugated boundary, Quart. Appl. Math. (to appear)

[9] H. Aref, Stirring by chaotic advection, J. Fluid Mech. 143, 1-21 (1984)

[10] A. J. Lichtenberg and M. A. Lieberman, Regular and Stochastic Motion, Springer-Verlag, New York, 1983

[11] H. Aref and S. Balachandar, Chaotic advection in a Stokes flow, Phys. Fluids 29, 3515-3521 (1986)

[12] J. Chaiken, R. Chevray, M. Tabor, and Q. M. Tan, Experimental study of Lagrangian turbulence in a Stokes flow, Proc. Roy. Soc. London. Ser. A 408, 165-174 (1986)

[13] P. D. Swanson and J. M. Ottino, A comparative computational and experimental study of chaotic mixing of viscous fluids, J. Fluid Mech. 213, 227-249 (1990) 